30

\title{
World Wide Simulation Environment (WISE)
}

\section{Web-based simulation using three dimensional models for manufacturing and material handling}

Kazuo Morita, Koji Kawashima, Junya Saito, Yoshinori Naruko

Mitsui Engineering \& Shipbuilding Co., LTD.

Makuhari Techno Garden 1-3-D7, Nakase, Mihama-ku,

Chiba-City, Chiba 261, Japan

Phone +81-43-274-0321, Fax +81-43-274-0324

e-mail:kmorita@mes.co.jp,kkawshi@mes.co.jp

\section{Masahiro Okamoto}

Toyada Machine Works, LTD.

\section{Hiromasa Suzuki}

Department of Precision Machinery Engineering The University of Tokyo

\begin{abstract}
This paper describes a Web-based simulation using three-dimensional models for manufacturing and material handling. The goal of this study is to construct a simulation environment that builds a virtual machine representing real equipment and a virtual factory collecting the virtual machine through a global communication network and executes them. This simulation environment is called World Wide Simulation Environment(WISE). The simulation environment is built with a new object-oriented language and architecture.
\end{abstract}

In this paper, arguments about simulation in manufacturing are clarified in order to identify requirements for next-generation manufacturing simulation systems. Then, models that meet the requirements of this concept are reviewed and, where suitable, 
are proposed for WISE. Furthermore, a system for demonstrating a Web-based virtual manufacturing simulator system is described in order to verify the operability of the system.

\section{Keywords}

WWW(World Wide Web), simulation, object oriented technology, product model, INTERNET

\section{INTRODUCTION}

Under increasingly competitive conditions in manufacturing sector, process engineering requires drastic improvements in all process. Simulation-based design is a new approach which, at the design stage, aims at developing a new environment for design, creation and operation of virtual and real products and process, focused on increasing real product value and reducing life cycle cost.

The main object of such a manufacturing simulation is a virtual environment which can provide support, information and control during all parts of the production cycle. Furthermore, this environment has to support internationalization and decentralization of the design and production activities, and simulation technology is very effective tool for this purpose. On the other hand, the tool is not good enough to support all parts of all production process. Therefore, to construct the next generation manufacturing system, a virtual environment is required that will support the life cycle of product, ranging from the planning to production, maintenance and reuse.

The goal of this study is to construct an environment that simulates a virtual manufacturing through a global communication network. This simulation environment is called the World Wide Simulation Environment(WISE), and it is built with a new object-oriented language and architecture called Umbel.")

In this project, physically meaningful models or product models with behavior, are built in a distributed environment and distributed throughout a network. The models are made to represent the function, behavior, and performance of real parts and manufacturing equipment ( such as robots, NC machines, AGVs, AS/RS, controllers, sensors) and human beings, by giving behaviors to three dimensional CAD models which represent real parts and equipment and correspond to the "behavioral product catalogs" of real equipment.

Users can thereby collect such pieces of equipment through a wide-area network and construct a manufacturing system. Since the physical model through the communication networks is simulated as a virtual machine representing a real equipment, so that a virtual plant built on such a network represents a real manufacturing plant and can be utilized to control an actual factory. The virtual 
factory can communicate through a network and support collaborative engineering among the enterprises and facilities distributed worldwide. So, We call this environment as WISE.

\section{WISE MODEL AND FUNDAMENTAL STUCTURE}

\subsection{WISE ARCHITECTURE.}

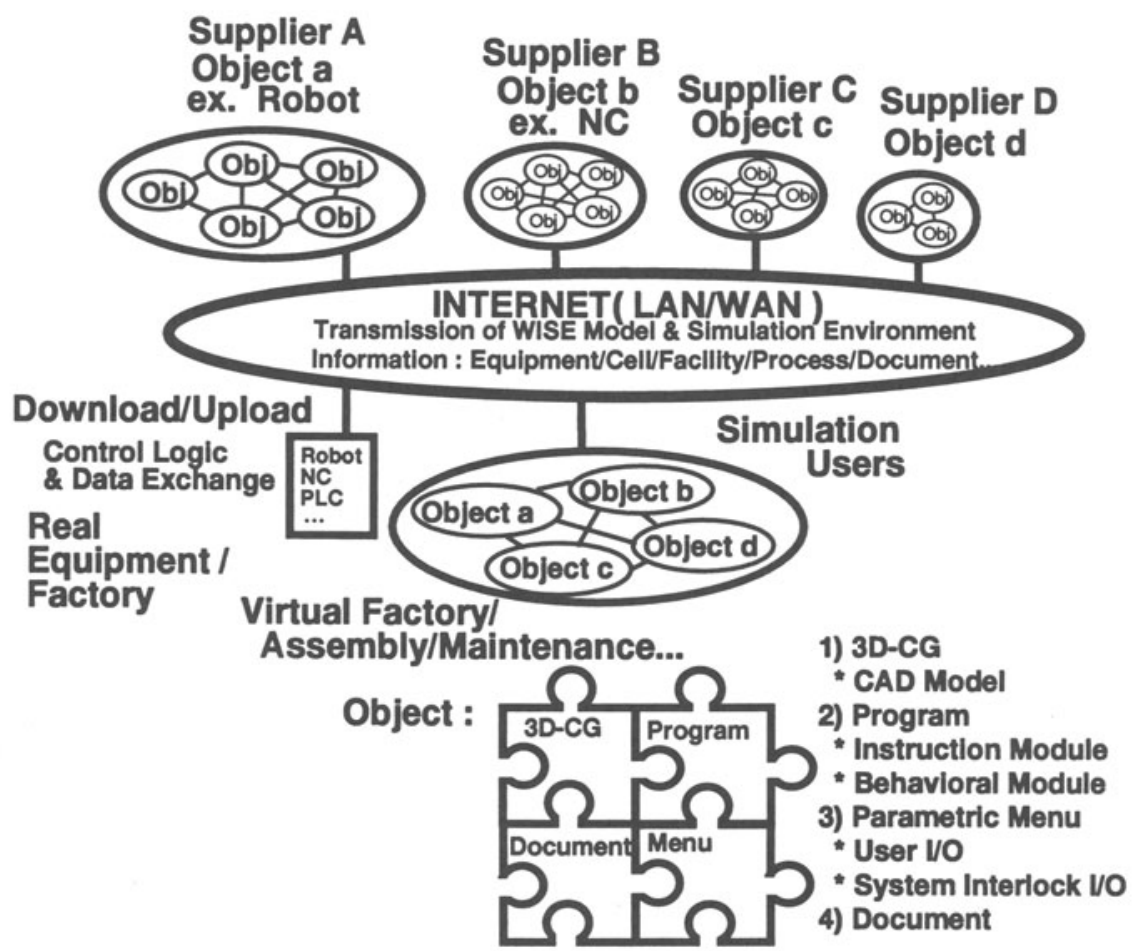

Figure1 World Wide Simulation Environment(WISE) Architecture

The WISE networking based simulation architecture is shown in Figure 1. The system has to distribute electronic catalogs to the client side where factory product engineer can construct a virtual factory in client-sever environment via INTERNET. The communication environment can transfer the WISE model by designating the URL address in the WWW browser based on the HTTP protocol. The WISE model is constructed as an integrated file which is taken in by designating the URL address and constructed by a three-dimensional model, the objects oriented program showing it's behavior and HTML multi-documents for GUI. In such a virtual factory 
construction environment, a factory engineer can execute the simulation by communicating via HTML multi-documents after acquiring the electric catalog through the INTERNET.

This simulation environment is investigated from the language to application level using Inflorescence, Inc.'s Umbel.

\subsection{STANDARD MODEL OF WISE}

The Basic requirements necessary for WISE model are defined below

\section{(1) Configuration of simulation model}

\section{a) Basic WISE model requirements}

Software language environment requires:

- The creation of a three dimensional model in order to represent the product model of each machine.

- Hierarchical programming, which is handled as a unit, and creating a new program combining the existing program, and the object oriented language which can create a reusable object encapsulating data and function.

- A concurrent environment with a scheduler function, for control the autonomous behavior of each machine object, and the ability to define and communicate by signal to control behavior.

- Interpreter and compiler language for creating the program flexibly and quickly.

- Transmission of the electronic catalog via the INTERNET by designating the URL address, accessing to the programs on the Web which implement the programs in HTML function and execute the simulation using these programs.

- An animation function that operates while simulation is running to enable the products engineer to easily understand the behavior of the virtual machine and activity of the virtual factory.

\section{b) WISE model and its basic configuration}

On the basis of these requirements, a simulation model is proposed that can support a plant life cycle from conceptualization to operation. The proposed model is a physical model that considers equipment and operations in a real plant from the software engineering viewpoint. The proposed model is not abstract mathematical model like a stochastic queuing network model in which entities representing products flow in a network between nodes which correspond to equipment.

An example of a detailed robot object for a WISE model consisting of links and each servo motor for the links is shown in Figure 2. This equipment class represents the physical model of the equipment. For example, if a servo motor is attached to a 
link, the motor rotates with the link. This robot class consists of the inverse kinematics and the synchronized function which represents real behavior. The relationship between objects are shown in Figure $3^{2)}$ and represent dependency between equipment, along with standard job( behavior) and parts. First, equipment is conceived as an abstracted model which performs processing with the lapse of time. Then, a physical model based on the abstract model is derived. This physical model is a three-dimensional model with actual behavior and is branched into robot model and so on. In this way, a simulation environment is constructed that is consisted through all stage from conceptual design to a detail design reflecting actual operational behavior. One of jobs shows the relationship between current jobs and next jobs.

\begin{tabular}{|l|}
\hline Robot \\
\hline Shape: Array of link figure \\
Motor. Array of each axis motor \\
Number of links: \\
D-H Parameters(Link parameters) \\
Solution of inverse kinematics \\
Home position angle of motors \\
Ready position angle of motors \\
Initial position of motors \\
Shape of Tool \\
\hline Function setting the target angle \\
Synctronized function for motor motion \\
Inverse kinematics function \\
Task for moving to target position \\
Task for moving to home position \\
Task for moving to Ready position \\
\hline
\end{tabular}

Figure2 Robot class

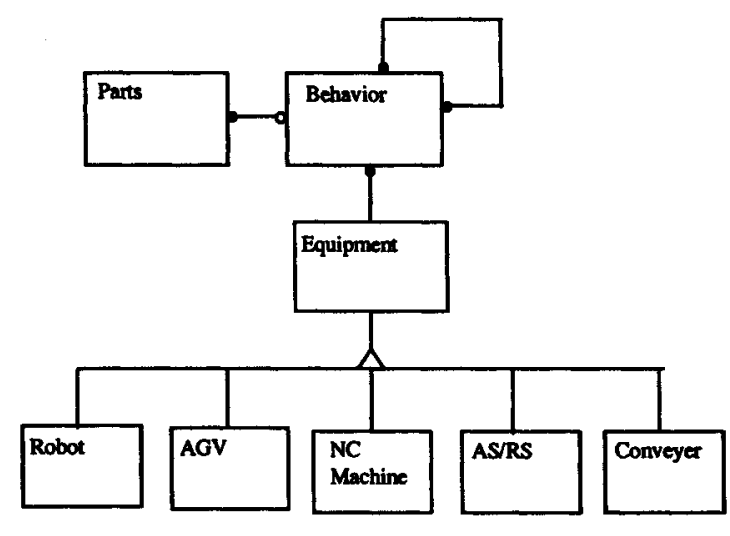

Figure3 Relationships between objects

Each individual object is a primitive object within the whole system. Therefore, behaviors can be defined as combinations of the methods of these primitive objects. In other words, all behavior is expressed by primitive methods in the same manner as real equipment is composed of individual components. This is the reverse to of complicated behavior being divided into simple behaviors and then further into primitive behaviors. In each behavior, signals are embedded in such a way that a behavior can cause other behaviors in a chain. A control object can perform centralized or parallel control. Consequently:

- Product model and Sequential behaviors can be defined as 1:n.

- Individual behavior can be combined arbitrarily sequences by signals.

- Each process is started up by signal to drive any objects.

Here, a process is an aggregate of behavior sequences and shows collaborative behavior. As a result, the user can have an environment that allows selection of arbitrary behaviors and processes against defined figures, and along with immediate execute. 


\section{WWW BROWSER-BASED DEMONSTRATION SYSTEM}

First, Robots, NC machines and AGV are constructed as each electronic catalog to simulate a factory. Next, a factory cell is constructed in local site after loading each electronic catalog, and the behavior of the machine is taught by the product engineer, and the simulation of cell is executed.

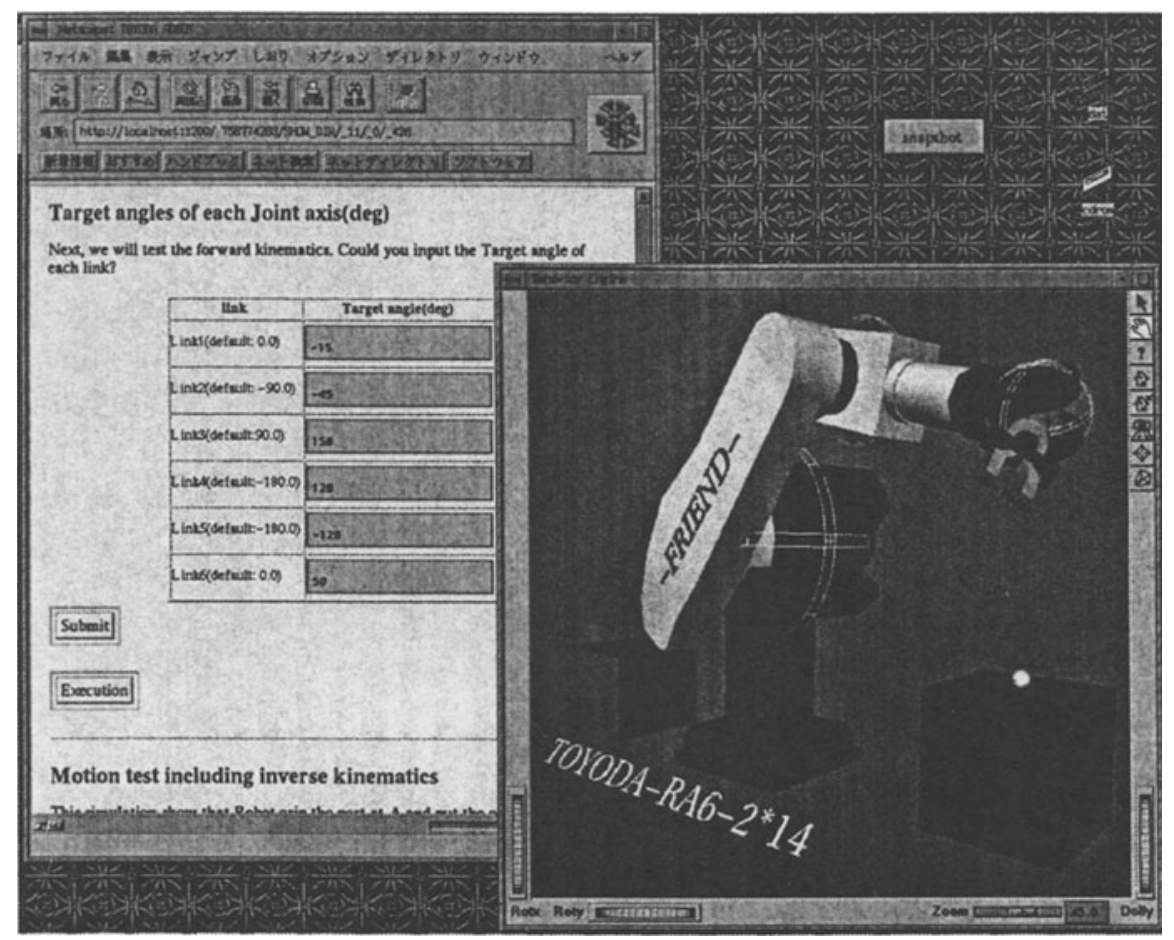

Figure 4 The electronic catalog of robot

The electronic catalog consists of equipment class, behavior programs, three dimensional models by Open Inventor, and an input panel for teaching and executing behaviors by HTML. It can communicate through INTERNET.

\section{(1) Robot}

An electronic catalog of the robot with six joints is shown in Figure 4. The input panel divides into three areas.

(a) Definition of acceleration, velocity and deceleration of each servo motor.

(b) Execution of forward kinematics by setting the target angle.

(c) Execution of a behavior series solving the inverse kinematics for the target position and gripping the target part. 


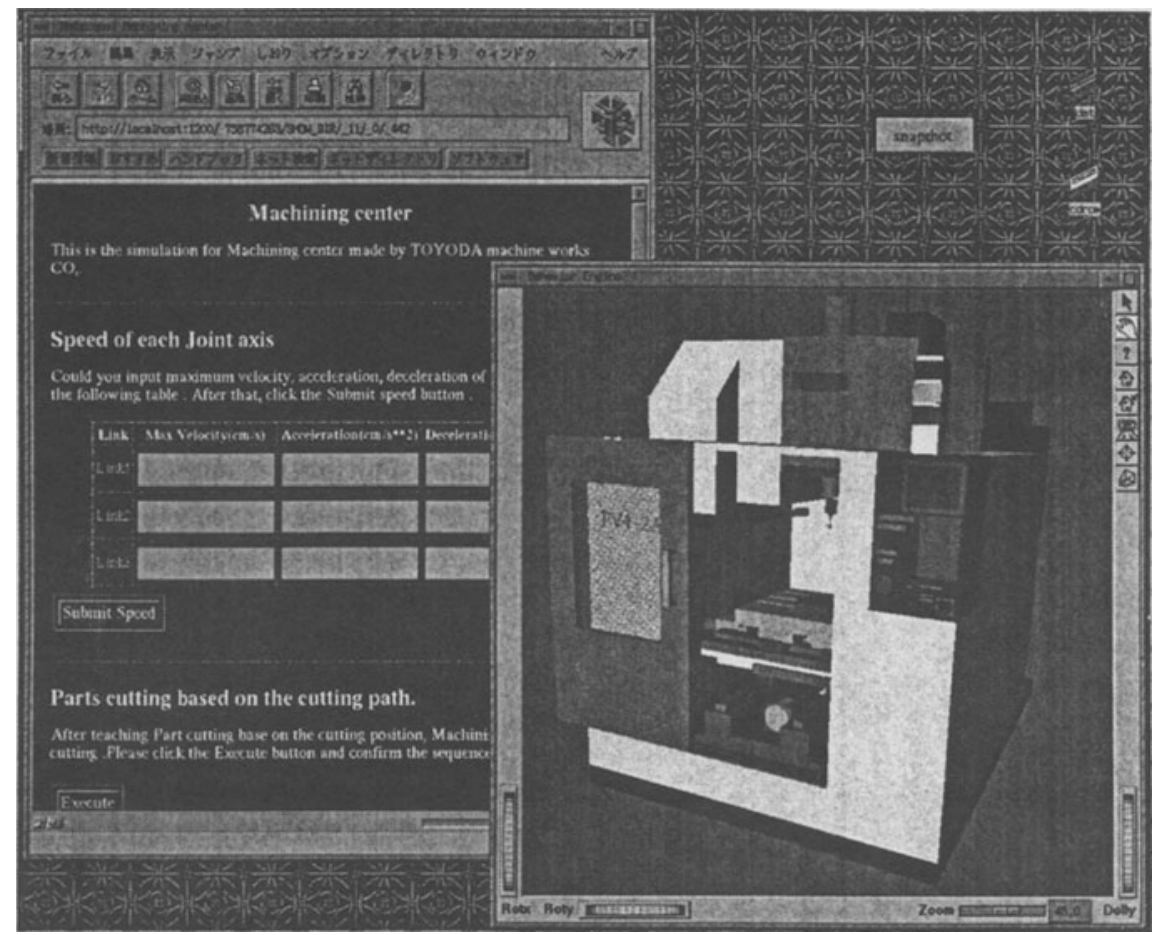

Figure 5 The electronic catalog of NC machine

(2) NC machine

The electronic catalog of a three axis NC machine is shown in Figure 5. The input panel divides into two parts.

(a) Definition of acceleration, velocity, and deceleration of each axis.

(b) Execution of a behavior series required for cutting a part based on inverse kinematics for cutting position on the part.

(3) AGV

The input panel is divides into two areas.

(a) Definition of translation acceleration, velocity, and velocity on the curved rail.

(b) Execution of behavior moving on guided path. 
(4) FA cell simulation

The FA cell is constructed by robot, NC machine and AGV electronic catalogs. In this process a product engineer constructs an FA cell after evaluating the performance of each machine using the electronic catalog for each piece of equipment. First, the product engineer loads the file integrating the machine model and behavior program of each machine, then teaches the behavior using an HTML menu and executes the simulation concurrently. By these process, the virtual simulation shown in Figure 6 can execute while taking the real performance of each machine and activity of the factory into consideration.

The simulation sequence shown in Figure 7 represent relationships among dynamic behaviors in an expansion of IDEF ${ }^{3)}$. The part flow is shown by a doted line, and the control signal flow is shown by a solid line. This is representative job flows expressed by a chain of behaviors. Such behavior divided into primitive behavior elements ( motion). The junction in IDEF3 can be also executed. Each behavior represents the autonomous behavior which is encapsulated object with input and output control ports. The connection between the input and output ports defines the simulation sequence. All behavior and related signal shows in Figure 8.

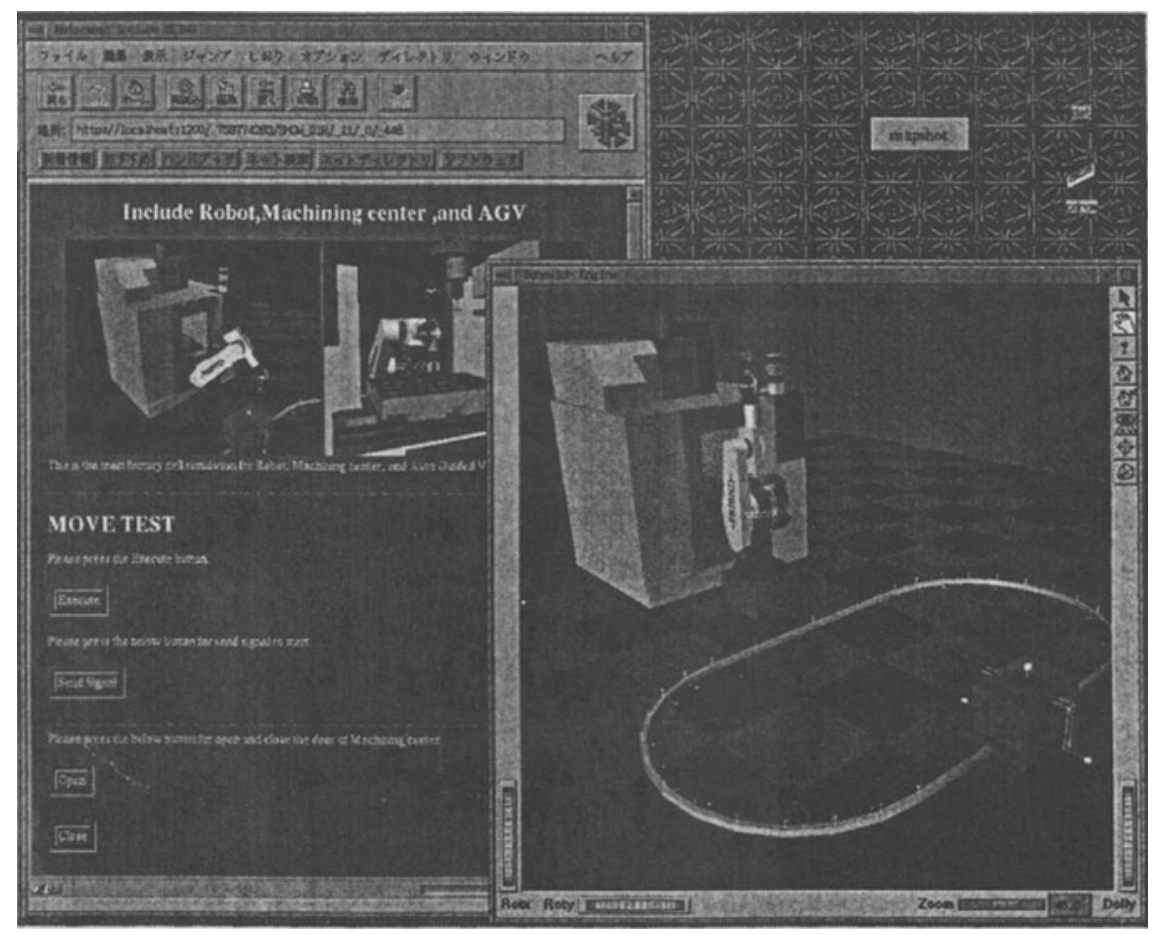

Figure 6 Executing simulation of FA cell 


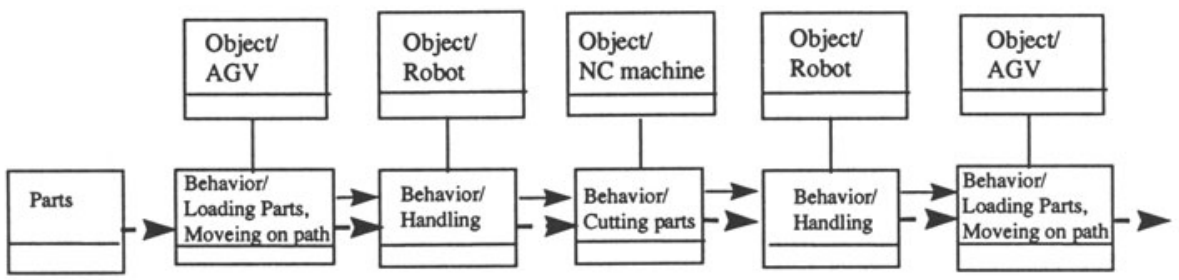

Figure 7 Simulation sequence by IDEF3

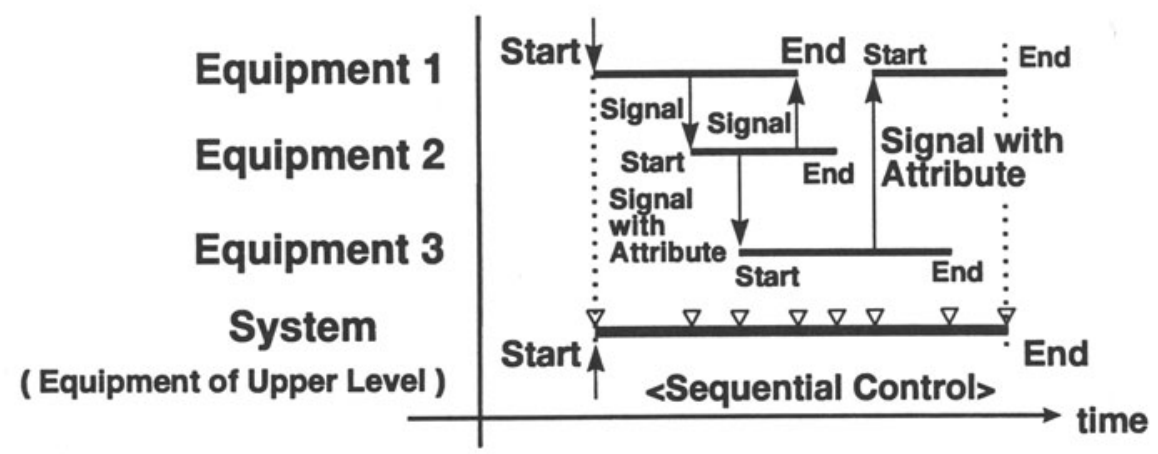

Figure 8 Signal interaction among behaviors

Definition of manufacturing Scenario/Process

Concurrent process Control using Message among Objects

(Autonomous Sequential and Central Control )

\section{CONCLUSION}

A networking based simulation architecture and its configuration for a communication model (WISE model ) that can exactly represent real equipment in the software is presented. A WWW browser-based FA simulation demonstration system is then developed and the workability of the principle demonstrated and validated.

The purpose of the study is to create such an environment that can execute a virtual plant simulation in accordance with a scenario through INTERNET. The simulation follows a procedure in which the parts or equipment manufacturer provides its own WISE model as a "product catalog" that behaves autonomously, and the user 
collects catalogs and assembles them together to construct an actual plant by using interlock signal. Furthermore, the virtual plant itself provides, through communication, wide-area collaborative engineering support environment in order to support the virtual enterprise.

At Mitsui Engineering \& Shipbuilding Co., Ltd., this concept will be used as the next step for the virtual operation training and remote maintenance etc. through INTERNET in order to achieve the virtual enterprise.

Finally, this research and development is being implemented as a part of Globman21 project under the umbrella of International IMS program.

\section{REFERENCES}

1) Chris Goad, "A Language-Level Attack on Compositional Simulation" http://www.inflor.com/compsim.html

2) James Rumbaugh, et al.(1991) "Object-Oriented modeling and Design", Prentice-Hall International, Inc.

3) Richard J. Mayer, et al. (1992) "Information Integration For Concurrent Engineering(IICE) IDEF3 Process Description Capture Method Report", Knowledge Based System, Inc.

\section{BIOGRAPHY}

Kazuo Morita is an Assistant Manager for Advanced Machine \& System Div. of Mitsui Engineering \& Shipbuilding Co., Ltd. He received a Bachelor's degree in Mechanical Engineering and a Master of Engineering from Nihon University. He is developing a new object oriented simulator for manufacturing and material handling. 